\title{
Comunicación y análisis socio-técnico: sentidos sobre la identidad local en la provincia de Misiones, Argentina
}

\section{Communication and socio-technical analysis: senses on the local identity in the province of Misiones, Argentina}

\author{
María Itatí Rodríguez ${ }^{1}$ \\ itatirodriguez@yahoo.com.ar \\ https://orcid.org/0000-0002-4299-8003
}

Recibido: 07/12/2020, Aceptado: 26/03/2021

\begin{abstract}
RESUMEN
La provincia de Misiones es una de las más jóvenes provincias argentinas. Su soberanía como provincia argentina data de mediados del siglo XX. Previamente su territorio ha pasado por varias denominaciones y autoridades. Para lograr su autonomía determinados grupos sociales relevantes tuvieron que construir distintos dispositivos para avalar una identidad e historia propia. En la actualidad podemos identificar procesos de producción de sentidos sobre una identidad local relacionados con políticas del gobierno provincial. En este artículo nos proponemos poner en diálogo los aportes de los estudios de comunicación con algunas herramientas del análisis socio-técnico, las cuales ofrecen al analista nuevas aperturas de lecturas para comprender y complejizar la mirada sobre nuestros objetos e interrogantes. Nos proponemos analizar las distintas alianzas socio-técnicas puestas en funcionamiento para la producción de una identidad local, es decir, reconstruir la trayectoria socio-técnica del artefacto. En este contexto, en la provincia de Misiones, mediante un conjunto de acciones (cognitivas, artefactuales y práxicas) realizadas por determinados grupos sociales relevantes (el ejecutivo provincial, los medios de comunicación, los investigadores e historiadores, los agentes escolares, etc.) buscan instalar determinados sentidos de una identidad local, con fuerte hincapié en una figura histórica -Andrés Guacaurarí y Artigas- mediante la producción y construcción de determinados artefactos, procesos, productos y organizaciones. Para ello en un primer momento ofrecemos una introducción a la temática, luego nuestras perspectivas teórico-metodológicas. En tercer lugar, reconstruimos dos fases para comenzar a identificar la trayectoria socio-técnica de estos artefactos. Realizamos un recorte cronológico -no riguroso sino orientativo- en los cuales estas configuraciones contemporáneas se observan con más fuerza: un periodo que va desde 1992 al año 2000, y otro que inicia en 2010 hasta el 2015. Finalmente ofrecemos algunas consideraciones finales a modo de discusión.
\end{abstract}

Palabras clave: comunicación, análisis sociotécnico, tecnología, identidad, misioneridad

${ }^{1}$ Universidad Nacional de Misiones, Argentina 


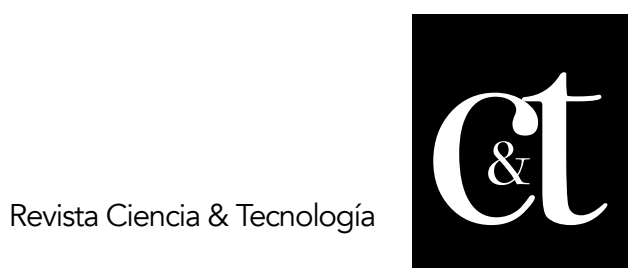

No. 30, 30 de abril de 2021

ISSN impreso: 1390 - 6321

ISSN online: 2661 - 6734

\begin{abstract}
Misiones province is one of the youngest Argentine provinces. Its sovereignty as Argentine Province dates from the mid 20th century. Previously its territory has gone through several names and authorities, Autonomy selected relevant social groups had to build different devices to endorse an identity and its own history. We can now identify production processes on a local identity senses related to policies of the provincial government.

In this article we intend to put into dialogue contributions from studies of communication with some socio-technical analysis tools, Analyst which offer new openings of readings to understand and complicate the look on our objects and questions. We propose to analyze the different socio-technical alliances put into operation for the production of a local identity, that is, to reconstruct the socio-technical trajectory of the artifact. In this context, in the province of Misiones, through a set of actions (cognitive, artifactual and praxical) carried out by certain relevant social groups (the provincial executive, the media, researchers and historians, school agents, etc.) they seek to install certain senses of a local identity, with a strong emphasis on a historical figure - Andrés Guacaurarí y Artigas - through the production and construction of certain artifacts, processes, products and organizations. To do this we initially offer an introduction to the subject, then our theoretical-methodological perspectives. Third, we reconstruct two phases to begin to identify the socio-technical trajectory of these artifacts. We made a chronological cut -not rigorous but indicative- in which these contemporary configurations are observed with more force: a period that goes from 1992 to the year 2000, and another that starts in 2010 until 2015. Finally we offer some final considerations by way of discussion.
\end{abstract}

Keywords: comunication, socio-technical analysis, technology, identity, misioneridad

\title{
Introducción
}

La provincia de Misiones conforma una de las 23 provincias argentinas y se encuentra ubicada en su región noroeste, conocida por su geografía como región mesopotámica. Su población, según el censo nacional del 2010, superó al millón de habitantes ( 1.101 .593 hab.) representando el $2.74 \%$ de la población total argentina. En el ámbito turístico, es reconocida mundialmente por ser la provincia de las "Cataratas del Iguazú", una de las siete maravillas naturales del mundo ${ }^{2}$. Otra de las características que se destaca de la provincia es su gran extensión de frontera internacional, un total de $80 \%$ : limita al oeste

2 En 2011 las Cataratas del Iguazú fueron elegidas nuevamente como una de las 7 maravillas naturales mediante el concurso de la Fundación "New Seven Wonders of Nature". Los otros seis sitios naturales elegidos son la Selva Amazónica (que involucra a ocho países sudamericanos); la Bahía Halong, en Vietnam; la Isla Jeju, en Corea del Sur; la Isla Komodo, en Indonesia; el Río Subterráneo de Puerto Princesa, en Filipinas, y la Montaña de la Mesa, en Sudáfrica. Más información: maravillas-del-mundo

Rodríguez. Comunicación y análisis socio-técnico: sentidos sobre la identidad local en la provincia de Misiones, Argentina 


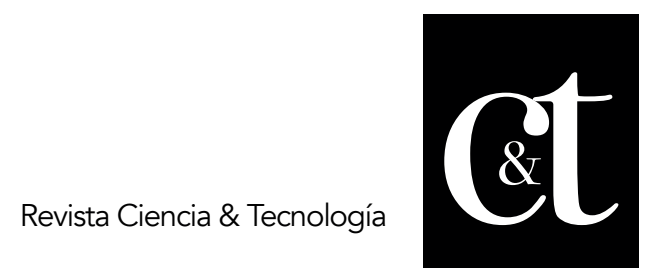

No. 30, 30 de abril de 2021

ISSN impreso: 1390 - 6321

ISSN online: 2661 - 6734

con Paraguay; al este, norte y sur con Brasil; y al suroeste limita con la provincia argentina de Corrientes.

Gran parte del territorio que actualmente ocupa la provincia de Misiones ha pasado por varias denominaciones y de depender de varias autoridades hasta lograr su soberanía provincial a mediados del siglo XX. Luego de la expulsión de los padres jesuitas de la Compañía de Jesús en 1778 se dio inicio a la Provincia Guaranítica de Misiones, una provincia hispánica con un gobernador que dependía de Buenos Aires. Luego de finalizar la Guerra de la Triple Alianza (1865-1870) fue formalmente anexada a la órbita de la provincia de Corrientes, la cual ejerció el dominio y usufructo de sus riquezas naturales hasta 1884 cuando se sancionó la Ley N. 1532 de Organización de los Territorios Nacionales donde se le designó a Misiones con esta categoría. En este momento dejaría de depender institucionalmente de la provincia vecina de Corrientes, pasando sus autoridades a ser designadas nuevamente por el Gobierno Federal, sin la posibilidad que sus ciudadanos puedan elegir a sus gobernantes.

Recién en 1953 Misiones se consolida como provincia argentina por Ley Nacional 14.294, un paso más en la producción de su historia. Este reconocimiento implicó también la necesidad de construir distintos dispositivos para la producción de una identidad e historia propia. Sin embargo, los procesos de construcción de distintos artefactos simbólicos para la producción de una historia provincial poseen larga data. Se fueron gestando previos a su provincialización en la década del 50 en el cual distintos grupos (intelectuales y políticos de la época) procuraban acceder a los derechos que implicaba ser una Provincia Argentina. Los movimientos por la provincialización de Misiones comienzan a gestarse en la década de 1920 integrada por parte de las elites locales; situación que cobra mayor impulso e institucionalidad cuando se crea la "Junta de Estudios Históricos de Misiones" en 1939 (avalada por la Academia Nacional de Historia).

Desde este momento, distintos grupos sociales relevantes "inventaron" una serie de artefactos para abandonar las limitaciones en término de derechos políticos y civiles que implicaba ser Territorio Nacional. Siguiendo a Jaquet (2005), la invención de "Misiones" como categoría identitaria se convirtió en la expresión tautológica de su propia obra: la misioneridad, un imaginario basado en la supuesta existencia de valores y virtudes del "ser misionero", que fue construido desde un discurso histórico centrado en la figura de un indio guaraní -Andrés Guacurarí y Artigas- como "prócer" o "héroe" local, en el contexto de reivindicación provincialista de la década de 1950. Andrés Guacurarí y Artigas fue uno de los más fieles colaboradores de José Gervasio Artigas, quien lo apadrinó y lo adoptó como hijo cuando éste se encomendó a la causa de la Liga de los Pueblos Libres. Liga de los Pueblos Libres o Liga Federal fue una confederación de provincias aliadas dentro de las Provincias Unidas del Río de la Plata, liderada por José Gervasio Artigas (1764-1850). La Liga fue constituida por las provincias de Córdoba, Corrientes, Entre Ríos, la Provincia Oriental, Santa Fe y los pueblos de Misiones. 


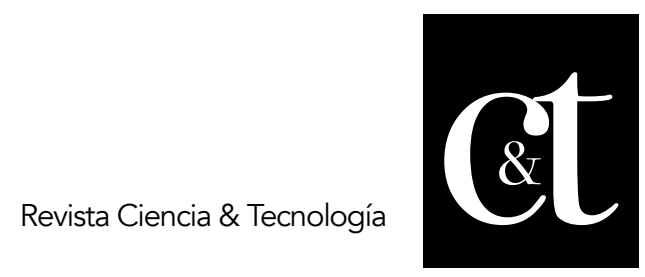

No. 30, 30 de abril de 2021

ISSN impreso: 1390 - 6321

ISSN online: 2661 - 6734

Felipe Pigna escribe que "para 1814, la popularidad de Artigas se había extendido a varias de las actuales provincias argentinas, afectadas, al igual que la Banda Oriental, por la política de libre comercio y puerto único, promovida por Buenos Aires, que arruinaba a los artesanos y campesinos del Interior $\langle\ldots\rangle$ Como Protector de la Liga, Artigas luchó junto con los jefes litoraleños contra el centralismo del Directorio. La liga formó una especie de mercado común regional en el que se protegía a los productores locales y se fomentaba la agricultura a través del reparto de tierras, animales y semillas. No pagaban impuestos las máquinas, los libros y las medicinas y derivaba el comercio del Litoral al puerto de Montevideo." http://www.elhistoriador.com.ar/biografias/a/artigas.php. Más conocido como "Andresito" denominado así a causa de su baja estatura (rasgo común entre la gran mayoría de los pueblos americanos) gobernó entre 1811 y 1822 la Provincia Grande de las Misiones, siendo elegido como Comandante.

El presente trabajo se inscribe en el marco de una investigación doctoral en comunicación (Rodríguez, 2016). En este artículo nos proponemos poner en diálogo los aportes de los estudios de comunicación con algunas herramientas del análisis socio-técnico, las cuales ofrecen al analista nuevas aperturas de lecturas para comprender y complejizar la mirada sobre nuestros objetos e interrogantes. Para ello proponemos analizar las distintas alianzas sociotécnicas puestas en funcionamiento para la producción de una identidad local, es decir, reconstruir la trayectoria socio-técnica del artefacto. En este contexto, en la provincia de Misiones, mediante un conjunto de acciones (cognitivas, artefactuales y práxicas) realizadas por determinados grupos sociales relevantes (el ejecutivo provincial, los medios de comunicación, los investigadores e historiadores, los agentes escolares, etc.) buscan instalar determinados sentidos de una identidad local, con fuerte hincapié en una figura histórica -Andrés Guacaurarí y Artigas- mediante la producción y construcción de determinados artefactos, procesos, productos y organizaciones. En este contexto proponemos dos fases para comenzar a identificar la trayectoria socio-técnica como co-construcción de determinados procesos organizacionales para la conformación de una identidad local. Realizamos un recorte cronológico -no riguroso sino orientativo- en los cuales estas configuraciones contemporáneas se observan con más fuerza: un periodo que va desde 1992 al año 2000, y otro que inicia en 2010 hasta el 2015.

\section{Metodología}

En el marco de una tesis doctoral en comunicación, en un sentido amplio, la investigación de la autora (Rodríguez 2016) tuvo como objetivo indagar sobre los procesos actuales de construcción y producción de identidades colectivas histórico-políticas (nacionales y locales) en relación a los mecanismos de memoria presentes en las efemérides y actos escolares de escuelas primarias públicas de la provincia de Misiones. Los estudios en comunicación hacen referencia a "complejos entramados históricos, institucionales e intersubjetivos que subyacen en la producción social de sentido, y no simplemente a los mecanismos, mediáticos o no, de producción, circulación y apropiación de 'mensajes'" (Fuentes-Navarro 2008: 154).

Rodríguez. Comunicación y análisis socio-técnico: sentidos sobre la identidad local en la provincia de Misiones, Argentina 


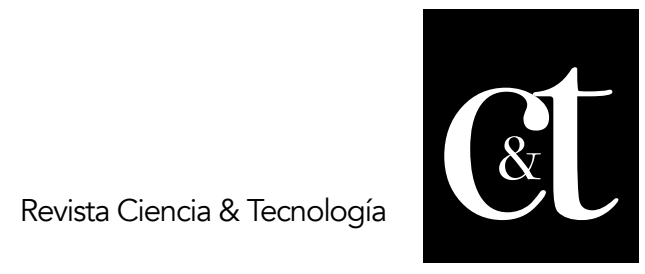

No. 30,30 de abril de 2021

ISSN impreso: 1390 - 6321

ISSN online: 2661 - 6734

Ubicándonos en una línea de pensamiento latinoamericano de investigadores de la comunicación, estos estudios apuntaron a "trascender el aspecto estrictamente técnico y del desarrollo de habilidades tanto de elementos discursivos como de los medios, para ubicarlo sobre todo en el espacio de las relaciones entre sujetos, enmarcados en contextos sociales y culturales" (Uranga 2007: 4). En esta ubicación política, histórica, geográfica, los estudios de comunicación latinoamericanos se traducen en un nuevo modo de relación con y desde las disciplinas sociales. Martín-Barbero (2002) advierte que estos procesos no escapan de recelos y malentendidos. Los estudios en comunicación se definen "más que por recurrencias temáticas o préstamos metodológicos por apropiaciones: trabajan procesos y dimensiones que incorporan preguntas y saberes históricos, antropológicos, estéticos" (Martín-Barbero 2002: 216). De esta manera, incorpora las posibilidades del trabajo desde la transdisciplinaridad, que no implica la disolución de sus objetos en las de otras disciplinas sociales, sino la "construcción de las articulaciones -mediaciones e intertextualidades- que hacen su especificidad" (Martín-Barbero 2002: 217) ${ }^{3}$.

Esta apertura de los estudios de comunicación nos permite continuar las tramas de lecturas de nuestro objeto, ampliar nuestros interrogantes, enriquecer miradas. Proponemos una lectura desde algunas herramientas de la perspectiva del análisis sociotécnico en el marco de los estudios sociales de la tecnología (Thomas \& Buch 2008). En este sentido, entendemos a la tecnología como una construcción social que "forma parte de un tejido sin costuras de la sociedad, la política y la economía" (Thomas 2008: 220). Siguiendo a Hughes (2008) los sistemas tecnológicos contienen componentes destinados a resolver problemas entremezclados, complejos. Entre sus componentes se encuentran artefactos técnicos, organizaciones, componente catalogados usualmente como científicos (libros, artículos, sistema de enseñanza universitaria, programas de investigación), artefactos legislativos. En este sentido podemos entender a todos los procesos, actores, grupos sociales relevantes y artefactos involucrados en la producción de sentidos para la configuración de una identidad local como complejos sistemas tecnológicos.

De esta manera, cada artefacto funciona como un componente del sistema e interactúa con otros artefactos, los cuales contribuyen directamente - a través de otros componentes a una meta común. Los artefactos son socialmente construidos y son diseñados para satisfacer diferentes criterios. Por otra parte, los grupos sociales relevantes constituyen a los artefactos, éstos le atribuyen distintos sentidos a partir de multiplicidad de visiones socialmente situadas. Asimismo, siguiendo a Thomas (2008) al referirnos a comenzar a trazar los mapas de una trayectoria sociotécnica hacemos referencia a un proceso de co-construcción de productos, procesos productivos y organizacionales e institucionales, relaciones usuario-productor, relaciones problemas-solución, racionalidades, políticas y estrategias de un actor determinado. Las trayectorias socio-técnicas nos permiten ordenar relaciones causales entre elementos heterogéneos en secuencias temporales (Thomas

3 "El análisis de la inserción de la comunicación en las prácticas sociales cotidianas se halla aún fuertemente condicionado por la diferenciación y especialización que la modernidad introdujo en la organización de lo social: diferenciación de las esferas y discursos de la ciencia, la moral y el arte, especialización de los espacios y las instituciones de lo político, lo económico, lo cultural" (MartínBarbero 2002: 221). 


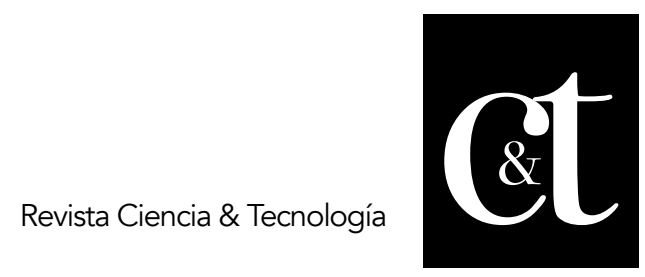

2008: 249). De esta manera, la apertura de las herramientas del análisis sociotécnico nos permite continuar complejizando las relaciones entre actores, artefactos, instituciones y relaciones de poder, es decir, la mirada sobre nuestro objeto como parte de un complejo tecnológico.

Finalmente, la perspectiva metodológica utilizada para nuestra investigación doctoral es la cualitativa. En este sentido comprendió las técnicas de diario de campo, observación participativa, entrevistas semi-estructuradas, registros audiovisuales. Estas instancias se realizaron en escuelas primarias públicas de las ciudades de Posadas y Puerto Iguazú de provincia de Misiones en el periodo 2013-2015. Por su parte, la búsqueda y la consulta de fuentes secundarias conforman otra de las estrategias de nuestro diseño metodológico. Estas últimas fueron las que colaboraron como el material de análisis de este trabajo. Los archivos de la Casa de Gobierno de la Provincia de Misiones, de la Subsecretaría de Cultura de la Provincia de Misiones, del Consejo General de Educación, de Institutos de Formación Docente de la provincia, de las Bibliotecas Populares de la ciudad de Posadas y de Puerto Iguazú, de la Facultad de Humanidades y Ciencias Sociales (UNaM), del Instituto Superior Antonio Ruiz de Montoya, y fundamentalmente, los archivos escolares de las organizaciones que conforman nuestro universo de estudio fueron espacios de recolección de datos de importante riqueza. Sumamos a las fuentes secundarias la búsqueda de información oficial en sitios web tales como: Ministerio de Educación de la Nación; Ministerio del Interior de la Nación; Ministerio de Cultura, Educación, Ciencia y Tecnología de la Provincia de Misiones; del Consejo General de Educación; así como también, sitios de noticias online y diarios impresos de edición provincial y nacional.

\section{Resultados y discusión}

Como primera fase podemos identificar un periodo que inicia a finales del siglo XX. En el marco del 40 Aniversario de la Provincialización de Misiones durante la década de 1990 el poder ejecutivo provincial estaba a cargo de Ramón Puerta (1991-1999) 4 del Partido Justicialista (alineado a lo que fue el gobierno del ex presidente argentino Carlos Menem). En esta fase podremos comenzar a observar la producción de una serie de artefactos en relación a la identidad local. Asimismo, podemos comenzar a organizar las relaciones que se establecieron entre los distintos elementos identificados: legislaciones, monumentos, sujetos sociales, organizaciones, etc.

En relación a la trayectoria socio-técnica, podemos identificar determinado carácter diacrónico, en el cual identificamos como punto de partida la puesta en escena de una tecnología legislativa como ser la instauración de la Ley N.3207/1995 que propone celebrar una "fiesta cívica" para recordar al héroe local. Sin embargo, antes aparece en escena como

${ }^{4}$ Federico Ramón Puerta nació en Apóstoles, Misiones, Argentina; político, ingeniero y empresario argentino perteneciente al Partido Justicialista. Ocupó el cargo de Gobernador de Misiones entre 1991 y 1999. Posteriormente, fue electo en diversas oportunidades como senador y diputado nacional. En diciembre de 2001, siendo senador alcanzó la Presidencia provisional del Senado y tras la renuncia del presidente Fernando de la Rúa estuvo a cargo del Poder Ejecutivo Nacional por unos días. En diciembre de 2015 fue designado por el presidente Mauricio Macri como Embajador argentino en España. 


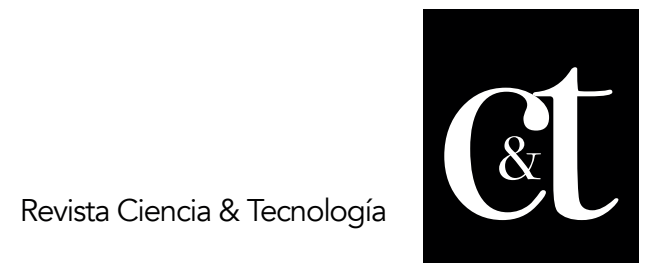

No. 30, 30 de abril de 2021

ISSN impreso: 1390 - 6321

ISSN online: 2661 - 6734

artefacto la Bandera de Misiones que fue instituida primero por decreto $\mathrm{N}$. 326/92 del Poder Ejecutivo provincial, luego en mayo de 1994 por la Cámara de Representantes de la Provincia de Misiones mediante la Ley N. 3102 (ahora Ley IV N.27 del Digesto Jurídico Provincial). Se fundamenta que los colores que componen la bandera (rojo, azul y blanco) son en homenaje a la Bandera de la Liga de los Pueblos Libres, liderada por José Gervasio Artigas, y padre adoptivo de Andresito. Esta legislación reglamenta su izamiento obligatorio en todos los establecimientos oficiales dependientes del Gobierno de la Provincia, las escuelas y actos públicos y estará colocada siempre a la izquierda de la Bandera Argentina (art.2). En este momento se tuvieron que construir (y/o agregar) pabellones provinciales en todas las organizaciones del Estado provincial (ministerios, secretarías, escuelas, etc.). Los portadores de esta Bandera serán los elegidos por sus pares como "mejores compañeros". El ritual de toma y traspaso de la Bandera Provincial se realiza una vez al año, y logran ser marcos de referencia importantes para aprender a recordar, a otorgarle un valor (lógico, ético y estético) a este artefacto. La incorporación de la Bandera de Misiones desde 1994, la elección de sus abanderados y escoltas es uno de los momentos estratégicos que ocupa un lugar central y se encuentra también reglamentado mediante un artefacto legislativo.

De esta manera, unos años después destacamos la instalación de la Fiesta cívica provincial por el Aniversario del nacimiento del prócer Don Andrés Guacurarí y Artigas - Día de la Bandera de Misiones" (Ley N.3207/1995, hoy Ley VI N.59). Esta celebración comenzó a incorporar esta fiesta como efeméride provincial todos los 30 de Noviembre, especialmente en la celebración de actos patrios en escuelas de la provincia (Rodríguez 2016). Fue durante el gobierno de Ramón Puerta -hoy opositor al gobierno provincial- que su figura se incluye de modo institucional. Esta efeméride recuerda el natalicio del héroe local. Cabe señalar que la figura de Andresito envuelve en parte misterio en relación a su nacimiento y muerte, sin lograr ser esclarecidos hasta la actualidad. Con respecto al año de nacimiento se discuten las fechas que van desde 1778 hasta 1790:

En cuanto a su origen, los conflictos fronterizos y la destrucción consiguiente de mucha documentación (incluyendo numerosos libros parroquiales de los pueblos) hace que no se pueda saber con precisión dónde y cuándo nació. Es probable que lo haya hecho en el pueblo de San Borga, aunque algunos historiadores sostienen que lo hizo en Santo Tomé. Tampoco se puede fijar la fecha; posiblemente vino al mundo un 30 de Noviembre, ya que, por su nombre de pila, resulta probable que lo haya hecho en el día de San Andrés. (Machón \& Cantero 2010: 66).

Luego, otro de los mecanismos aplicados para la construcción de la identidad local fue la instalación de la canción oficial de la provincia, una galopa escrita por el compositor Lucas Braulio Areco que aparece en el año 2000, cuando se impone primero como decreto (N. 813/2000) del gobernador Carlos Rovira, y luego como Ley 3910/2002 (en la actualidad LEY VI N.97 del Digesto Jurídico). Desde entonces es obligatoria su entonación en todos los actos oficiales del estado provincial y los actos de las escuelas públicas y privadas, a continuación del Himno Nacional Argentino (exceptuando el 20 de Junio, "Día de la Bandera Nacional Argentina"). 


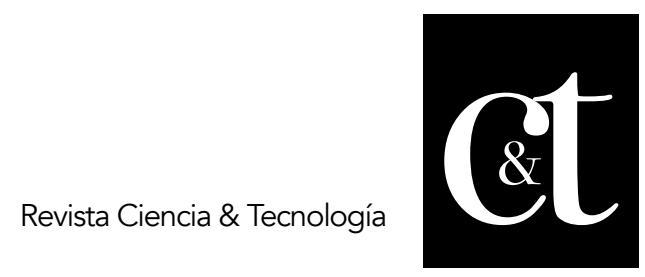

No. 30, 30 de abril de 2021

ISSN impreso: 1390 - 6321

ISSN online: 2661 - 6734

El artículo 2 dicta que "será entonada obligatoriamente después del Himno Nacional en todos los actos oficiales y escolares que se realicen en establecimientos dependientes del Gobierno de la Provincia". Asimismo, en el artículo 3 menciona que "será obligatorio su aprendizaje y difusión en toda la comunidad educativa de la Provincia, a la que se proveerá de la letra, grabaciones y partituras musicales pertinentes". Consideramos que su institucionalización como "canción oficial" forma parte de una estrategia por parte del Estado provincial para la conformación de una serie de dispositivos identitarios provinciales, en los que también se destacan la figura de Andrés Guacurarí y Artigas y la Bandera de Misiones.

Como menciona el proyecto de Ley que lo impulsa: "con la incorporación de la canción "Misionerita" como canción oficial de la provincia de Misiones se completa la trilogía de símbolos (a los que se suma el escudo y bandera, agregamos) que identifican muy especialmente a la provincia en el contexto de las provincias argentinas y además en toda esta región de América del Sur". Con respecto al ritmo, no fue ajena a las discusiones los procesos de su selección determinado ritmo, artista, canción por sobre otros también destacados en la provincia. En este sentido, el ritmo seleccionado para la canción oficial es la galopa misionera, género musical y de danza5

De la mano a la instalación de esta figura en el orden de lo público en el relato histórico, desde otros espacios, como ser el académico, se pueden observar distintos proyectos de investigación -desde la Universidad Nacional de Misiones, la Junta de Estudios Históricos de la provincia de Misiones u otros organismos de investigación- que abordan no solamente a la figura de Andresito desde una perspectiva histórica, sino otros que indagan sobre las tramas que presenta esta producción de una identidad propia. Destacamos aquí el trabajo del historiador Jorge Machón (1996) y Héctor Jaquet (1998) en esta fase.

Como segunda fase -periodo comprendido desde el 2008 al 2015- de modo sincrónico podemos pensar determinados cambios socio-técnicos, una serie de artefactos puestos a funcionar, y cómo éstos interactúan con otros procesos y actores. En este sentido, las decisiones políticas aplicadas desde el Partido Renovador 6 de la provincia de Misiones, la fuerte presencia de determinados actores, como ser los distintos representantes del ejecutivo provincial, nos permiten realizar un mapa de análisis más complejo. Asimismo, en relación a la trayectoria socio-técnica, identificamos desde una mirada diacrónica, secuencias temporales y que entran en diálogo con procesos anteriores: la

\footnotetext{
${ }^{5}$ Este estilo proviene del galop, una expresión llegada desde Francia. Es una danza animada, introducida a finales de los años 1820 a la sociedad parisina por la duquesa de Berry y popular en Viena, Berlín y Londres.

${ }^{6}$ Este partido fue electo en el ejecutivo provincial sucesivamente desde el 2003 hasta la actualidad. Gobernador Carlos Eduardo Rovira (1999-2007), en el primer periodo perteneció al Partido Justicialista impulsado por el anterior gobernador, Ramón Puerta. Para su segundo periodo fue el impulsor del partido "El Frente Renovador de la Concordia" en el cual fue candidato y electo gobernador; luego el ex gobernador Maurice Closs (2007-2015, dos periodos) y el gobernador Hugo Passalacqua (2015, actual).
} 


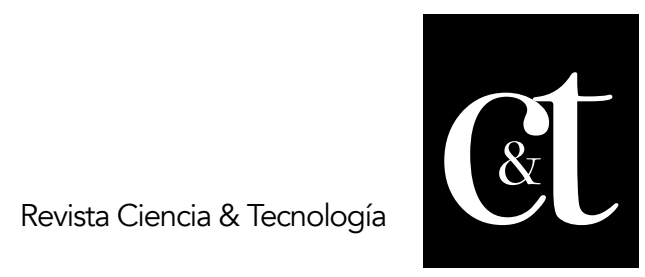

No. 30,30 de abril de 2021

ISSN impreso: 1390 - 6321

ISSN online: 2661 - 6734

apuesta de artefactos, procesos y organizaciones posteriores que se fueron organizando de modo causal y mediante elementos heterogéneos.

En este sentido, destacamos como marco de referencia de esta fase las celebraciones en el marco del Bicentenario de la Revolución de Mayo de 1810 y el 60 Aniversario de la provincialización de Misiones en 2013. Éste último se relaciona con una política específica del gobierno provincial, es decir, con el Partido Renovador de Misiones en el cual podríamos pensar que la causa "provincialista" y de Andresito pasó a formar parte de la base del discurso del partido político para, entre otras estrategias, instalar a la provincia en los relatos nacionales. Mediante distintos artefactos los sucesivos gobiernos provinciales impulsaron la producción de una batería de procesos, prácticas para la conformación de un relato provincialista, con fuerte impronta en Andresito Guacurarí como su mayor estandarte.

Desde los espacios universitarios de la región se desarrollaron distintos proyectos de investigación que indagaban sobre estos mecanismos para la producción de una historia local. Podemos volver a mencionar los avances del trabajo de Jorge Machón y Daniel Cantero $(2008,2010)$ desde la historia, de Héctor Jaquet (2005) desde la antropología, y de Marcelino García (2006a, 2006b) desde la semiótica y la comunicación. Por otra parte, apoyados por el gobierno provincial, también la Junta de Estudios Históricos de Misiones publica en 2010 en el marco de los festejos por el Bicentenario Argentino publica Miradas históricas sobre Misiones en el Bicentenario, que cuenta con artículos de investigadores de reconocimiento local, sobre temáticas referidas a la historia de Misiones. De esta manera, los festejos del Bicentenario de la "nación" coinciden como un encuentro, un diálogo con lo que los "misioneros" tienen para decir en este aniversario.

La escuela primaria y secundaria, de gestión pública o privada también ocupó un rol relevante en la puesta en escena de estos artefactos. Mediante la implementación del Calendario Escolar Permanente del Consejo General de Educación (Res. N.842/2006) que se presenta como un cronograma escolar básico y unificado para las escuelas y su desarrollo curricular pretendió ser un instrumento de trabajo que facilite la planificación, organización e implementación de la tarea del Sistema Educativo. En su "Normas de aplicación" dicta que "durante el homenaje patriótico estarán presentes la Bandera de Ceremonias y la Bandera Oficial de la Provincia de Misiones (excepto el 20 de junio en el cual no participará la Bandera de Misiones), de acuerdo con lo establecido en las normas sobre las características, tratamientos, usos de los símbolos nacionales y se cantará el Himno Nacional, la canción oficial de la Provincia de Misiones "Misionerita" y la canción correspondiente a dicha fecha". Asimismo, este Calendario incluye en la mayor jerarquía de las efemérides a conmemorar en el aula al 30 de Noviembre "Día de la Bandera de Misiones" y del "Prócer misionero Don Andrés Guacurarí y Artigas", tensionando nuevamente estos artefactos provinciales, con otros de alcance nacional, como ser la efeméride del 25 de Mayo "Día de la Revolución de Mayo de 1810". En Argentina el 25 de Mayo se recuerda la "Revolución de Mayo" en conmemoración a los sucesos ocurridos en 1810 en el cual los cabildantes del Cabildo de la ciudad de Buenos Aires reconocieron la autoridad de la Junta Revolucionaria y así se formó el Primer Gobierno Patrio. 


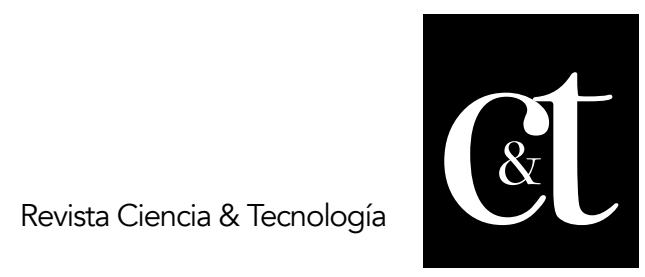

No. 30,30 de abril de 2021

ISSN impreso: 1390 - 6321

ISSN online: 2661 - 6734

Dicha efeméride se comienza a recordar a pocos días de lo sucedido y es incorporada de manera prácticamente simultánea a la implementación de la educación pública en Argentina a finales del Siglo XIX, a partir de la enseñanza en las materias de Historia, como en la celebración de las efemérides patrias. (Rodríguez 2016).

La legislación que marca con mayor fuerza este conjunto de alianzas socio-técnicas es la Ley VI N.155/12 que declara a Andrés Guacurarí y Artigas como prócer provincial logrando instalar su figura como un artefacto simbólico mediante distintas estrategias en la escena política, educativa, y social. Sin embargo, por esas luchas/disputas que tiene ( $\mathrm{n}$ ) la (s) memoria (s), podemos leerlo con mayor complejidad mediante el análisis socio-técnico propuesto- esta reglamentación lo vuelve a declarar prócer a Andresito: su figura se (re) inventa con un rótulo de mayor jerarquía. Recordamos que la Fiesta Cívica Provincial del día 30 de Noviembre (Ley N.3207/1995) lo instala como prócer misionero y propone recordar su natalicio. En esta oportunidad, la Cámara de Representantes de la provincia de Misiones -siendo su presidente el diputado provincial Carlos Rovira, mediante esta tecnología lo declara (nuevamente) prócer en el rótulo de "Comandante General" -olvidando quizá sutilmente una anterior declaración del mismo corte. El Frente Renovador lleva la "causa Andresito" como propia, como una bandera política.

En este contexto, la legislación incorpora distintos artefactos para la producción de una identidad local como ser la incorporación del "prócer misionero" en los libros de textos oficiales y no oficiales, en los programas educativos y museos y la construcción de un monumento en la costanera capitalina misionera. La obra fue realizada por el artista Gerónimo Rodríguez, quien además ha realizado la mayoría de las obras artísticas (murales, monumentos, estatuillas, etc.) en distintas localidades de la provincia, y por distintos acontecimientos, siempre en relación a la temática de la historia regional. De esta manera, el levantamiento del monumento al prócer local logro instalar de una manera singular e imponente, al "héroe" en uno de los centros de la vida urbana de la ciudad. ${ }^{7}$ En este contexto, la provincia de Corrientes erige un monumento a Andresito en la rotonda de la costanera Sur contruida por el colectivo de artistas "Cultura Vallese" e integrantes de la productora Payé Cine. ${ }^{8}$

En el marco de esta reglamentación se lanza el "Portal Andresito. El portal educativo de la provincia de Misiones", auspiciado también por la Cámara de Representantes de la provincia de Misiones, declarado de Interés provincial. Posee para consulta y descarga materiales (actividades, contenidos y juegos) para nivel inicial, primario, secundario y para estudiantes de Institutos de Formación Docente con respecto a la temática "Andresito". Éste cuenta con un "maletín de recursos" con aplicaciones de software libre para docentes y

7 Imágenes disponibles en el Facebook del diario MisionesOnLine: https://www.facebook.com/media/set/?set=a.10151615883492911.1073742035.103253332910\&typ $\mathrm{e}=3$

8 Más información: http://www.ellitoral.com.ar/es/articulo/313216/Construyen-la-imagen-deAndresito.

Rodríguez. Comunicación y análisis socio-técnico: sentidos sobre la identidad local en la provincia de Misiones, Argentina 


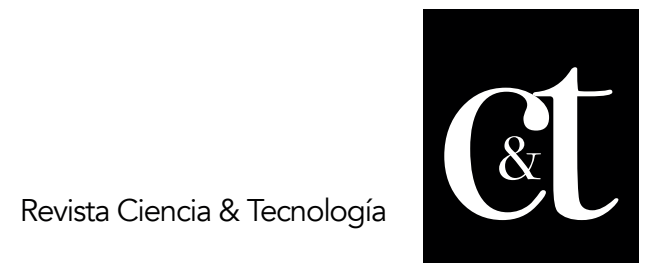

No. 30, 30 de abril de 2021

ISSN impreso: 1390 - 6321

ISSN online: 2661 - 6734

estudiantes. Y un espacio virtual para consultar sobre la vida, videos, campañas, leyes sobre Andresito. También podemos mencionar también la publicación de Atlas "Misiones 21", publicada por el Ministerio de Educación de la provincia en el 2008. En este sentido, el material consiste en un Atlas de 6 módulos: "Misiones, territorio y región", "Misiones y su gente", "Misiones y su historia", "Misiones y su naturaleza", "Misiones y sus áreas protegidas", "Misiones y sus paisajes rurales y urbanos".

En 2013, cuando Misiones cumple el 60 Aniversario de su Provincialización se realizan distintas conferencias, muestras, encuentros, charlas, actos en toda la provincia. Entre estas actividades destacamos la serie de conferencias de los historiadores Pacho O Donnel y Felipe Pigna durante el 2012 en la cual reivindican el lugar de la provincia en la "Historia Argentina". Desde agosto a diciembre de 2013 se realiza una Muestra sobre la Provincialización en el Centro del Conocimiento de la ciudad de Posadas ${ }^{9}$. Asimismo, en el marco de las reglamentaciones incorporadas por estos años, también podemos mencionar las que comprenden la designación del nombre del héroe a distintos espacios públicos. Por ejemplo, por Ley VI N.157, se designa en 2013 con el nombre de "Comandante General Andrés Guacurarí y Artigas" a las Rutas Provinciales N. 105, 1, 2, Acceso Sur y Bypass Arco-Garita de la ciudad de Posadas y Garupá.

A nivel nacional, podemos destacar varios procesos que dieron fuerza a esta trayectoria socio-técnica. En 2014, la ex presidenta argentina Cristina Fernández de Kirchner por Decreto 463/2014 lo promueve "al grado de General post mortem al Coronel D. Andrés Guacurarí y Artigas" (art.1). Asimismo, se instala una efeméride a nivel nacional para recordar este héroe provincial ahora declarado a nivel nacional. El 2 de julio se recuerda la Batalla de Apóstoles liderada por Andrés Guacurarí y Artigas en 1817, como el "Día de la Conmemoración y recuerdo de Don Andrés Guacurarí". Fue un enfrentamiento ocurrido el 2 de julio de 1817, en el marco de la invasión luso-brasileña en la cual el general portugués Francisco das Chagas Santos invadió las Misiones Orientales y luego el territorio que actualmente ocupa la ciudad de Apóstoles (Misiones), donde Andresito estableció su cuartel general. Santos fue derrotado por el héroe misionero. Mediante esta efeméride Andresito es declarado héroe nacional, como "tributo y reparación histórica por su contribución a la epopeya de la emancipación del continente americano", incorporándose al Calendario de efemérides nacional en 2014.

Previo a estas declaraciones, la figura de Andresito lograba aparecer en distintas oportunidades en la escena nacional de la mano de la ex presidenta de la Nación Argentina, ambas en junio de 2013. Señalamos dos casos que envuelven la causa "Andresito", hechos cubiertos por los medios de comunicación y ampliamente difundidos por las redes sociales. Un primer ejemplo, con el debate sobre los fondos atribuidos del Instituto Nacional de Cine y Artes Visuales (INCAA) a la realización de audiovisuales durante la presidencia de Fernández de Kirchner. En una conversación pública entre el realizador de "En busca del Comandante Andresito", el correntino Camilo

\footnotetext{
9 A su vez, el Diario "El Territorio" publica una Edición Especial con motivo del 60 Aniversario Disponible para consulta: http://www.territoriodigital.com/suplementodigital/Especial/ElTerritorio/88-Aniversario/Index.html
} 


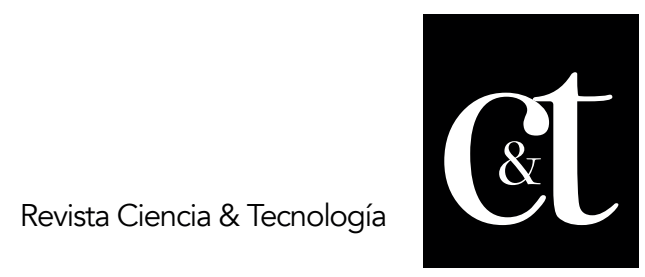

No. 30, 30 de abril de 2021

ISSN impreso: 1390 - 6321

ISSN online: 2661 - 6734

Gómez Montero de Payé Cine (documental que ganó un Concurso Federal del INCAA que posibilitó económicamente su realización) y la respuesta de la presidenta. $Y$ en otro caso, la revuelta ocasionada por una noticia de repercusión nacional que involucraba el cambio de nombre del Estadio del Club deportivo misionero "Crucero del Norte" que lleva el nombre de Andrés Guacurarí por el de la ex presidenta. ${ }^{10}$

Por su parte, en el Calendario de Efemérides Culturales promocionado por el Ministerio de Educación de la Nación aún no se registra dicha efeméride. Este Calendario de corte nacional sí señala e incorpora al 30 de Noviembre como el Día de la Amistad Argentino-Brasileña desde el 2004 (Decreto No 688 del Poder Ejecutivo Nacional) y el Día Nacional del Mate desde el 2015 Advertimos que las justificaciones de los días de ambas efemérides no entran en relación con la memoria del ahora prócer misionero ni de la bandera provincial.

Otro de los mecanismos que aparece en el centro de la "nación" es la inauguración del Monumento a las Cataratas del Iguazú y a Andresito en la Plaza de Misiones en la Ciudad Autónoma de Buenos Aires, en plena Avenida 9 de Julio. Como dato curioso, dicho emplazamiento debería haberse realizado casi cuarenta años atrás debido a que en 1975 por Decreto 1646 provincial se autoriza un contrato de obra con encargo para la realización de un busto de bronce con la figura del Comandante Andrés Guacurarí con el objetivo de ser exhibidos en el Paseo "Provincias Argentinas" en la avenida 9 de Julio de Capital Federal. "Andresito" fue considerado en esta legislación como un "destacado personaje dentro de la Historia Misionera". Dicho emplazamiento nunca se llegó a concretar. Más tarde, en 1986 se retoma el tema y por Ley 23.420/1986 durante el gobierno del ex presidente argentino Raúl Alfonsín se dispone la erección de un monumento a la memoria del comandante Andrés Guacurarí en dicha plaza porteña. En el artículo 3b, se propone que el Poder Ejecutivo, a través del Ministerio de Educación y Justicia de la Nación, difunda la vida y obra del comandante Andrés Guacurarí.

Sin embargo, todas estas legislaciones habían quedado truncas hasta el año 2013, que con un fuerte impulso del ex gobernador de la provincia de Misiones Maurice Closs al turismo, recrean una réplica de las Cataratas del Iguazú en pleno centro porteño. ¿Pero dónde quedó el monumento a Andresito? Varios medios de comunicación locales se hicieron eco de este olvido ${ }^{11}$ y luego de acalorados debates en la escena mediática y política local, se dispuso realizar dicho homenaje al indio guaraní en una plaza lindera. Así, en diciembre de 2013 con la presencia del Jefe de Gobierno Porteño (actual presidente de la Nación) Mauricio Macri y del gobernador de Misiones, inauguran dichos monumentos. Sin embargo, la obra expuesta ha tenido divergentes apreciaciones sobre su valor estético. La nota "Inventario de arte dudoso en el espacio público", publicada en Clarin.com (11-07-2015) firmada

\footnotetext{
10 Notas completas en: http://www.cfkargentina.com/

11 Por ejemplo, el diario "Primera Edición" en la sección Política y economía, titula "El monumento a Cataratas deja en el olvido el homenaje Andresito" (30/09/2012); luego en su sección Información General, "El monumento a Andresito se haría frente al de Cataratas" (11/10/2012).
} 


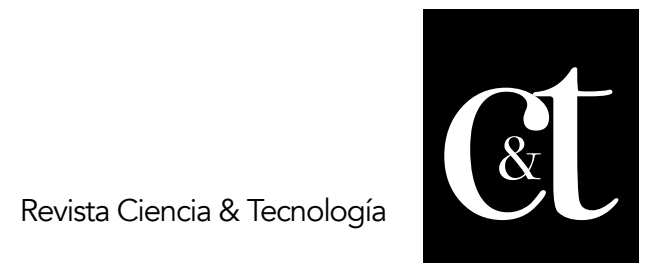

No. 30, 30 de abril de 2021

ISSN impreso: 1390 - 6321

ISSN online: 2661 - 6734

por el periodista March Mazzei que se refiere a la obra como: "el homenaje de Misiones al Comandante General Andrés Guacurarí y Artigas, que parece un personaje de ciencia ficción, entre el hombre de Hojalata de El Mago de Oz y Blender, el robot de Futurama. ${ }^{12}$

Finalmente, podemos mencionar al dispositivo denominado "la lanza de Andresito" que fue confeccionada por el reconocido orfebre argentino Carlos Daniel Pallarols quien es 70 generación de orfebres de Argentina, tiene una gran trayectoria reconocida en el país y el exterior, y desde hace 32 años realiza los bastones presidenciales de mando. El artista señala que "esta lanza recuerda la fuerza con la que él luchó por su tierra y con la que defendió sus ideales y su identidad. Símbolo de rectitud, justicia y heroísmo". El denominado bastón de mando "representa la voluntad de lucha de Andresito y sus indios, y fue realizada en homenaje al pensador y creador del Centro del Conocimiento, Ing. Carlos Rovira" según expreso el artista. La pieza está construida en plata 921 Argentina, el escudo de Misiones en oro 18 y la madera es anyico colorado, nativa de Misiones. ${ }^{13}$ De esta manera, el pasado 10 de diciembre de 2015, cuando el actual gobernador de la provincia de Misiones, Hugo Pasalacqua asume el mando, se realizó por primera vez el paso simbólico mediante "la lanza de Andresito" que consistió en que el gobernador saliente transfiere la titularidad del cargo ejecutivo provincial al gobernador entrante.

\section{Conclusiones}

En el presente artículo nos hemos propuesto entablar un diálogo entre los estudios de comunicación y algunas herramientas del análisis sociotécnico en relación a distintas alianzas socio-técnicas puestas en funcionamiento para la producción de una identidad local, es decir, reconstruir la trayectoria sociotécnica del artefacto. En este sentido, hemos observado cómo se configuraron distintos artefactos, actores, intereses políticos, económicos, culturales e históricos. Logramos identificar dos fases de la trayectoria sociotécnica en la cual se observan con fuerzas estos cruces, tensiones, producciones, es decir, como se co-construyen determinados procesos organizacionales para la conformación de una identidad local en la provincia de Misiones (Argentina). Observamos en este caso cómo determinados grupos relevantes y la puesta en funcionamiento de sistemas tecnológicos complejos pretenden instalar determinados sentidos de una identidad local, con fuerte hincapié en una figura histórica -Andrés Guacaurarí y Artigas- mediante la producción y construcción de determinados artefactos, procesos, productos y organizaciones. Estos artefactos ejercen agencia debido a que se encuentran integradas en dinámicas socio-técnicas. Esta capacidad de agencia es política, social, económica, y situada en determinado contexto de tiempo y espacio que hace posible su funcionamiento. En el caso presentado podremos observar cómo, desde la perspectiva socio-técnica, esta tecnología trama relaciones de poder, dominio y control sobre los sentidos en relación a la producción de una identidad local.

Como hemos desarrollado, fueron distintos los artefactos, procesos y actores que intervinieron en la construcción socio-técnica para la conformación

\footnotetext{
12 Nota disponible en: http://www.clarin.com/cultura/arte publico-estatua de gardelsirena del rio de la plata 0 1391861228.html

${ }^{13}$ Consultado en: http://portalandresito.gob.ar/contenidos/index.php/recorrido-virtual/2-general/40procer-misionero
} 


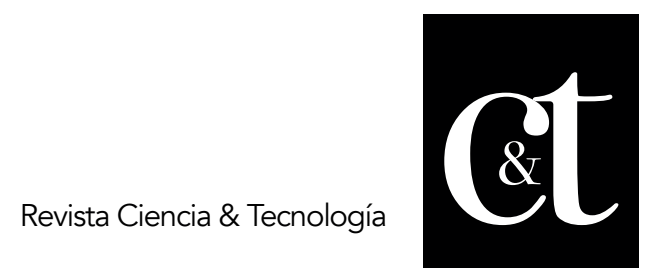

No. 30, 30 de abril de 2021

ISSN impreso: 1390 - 6321

ISSN online: 2661 - 6734

de una identidad local, centrados en la figura de Andrés Guacurarí y Artigas. Impulsados fuertemente por determinadas políticas del gobierno de turno, fueron distintos las estrategias y los objetivos propuestos. La mirada puesta desde lo social, lo tecnológico, lo político y lo cultural atraviesa todo el análisis, y es identificable con fuerza en cada fase. Históricamente situadas, responden a los interrogantes e intereses de determinado grupo de poder. En ambas fases identificamos una resignificación de tecnologías debido a que se han realizados operaciones mediante las cuales se han reutilizado tecnologías previamente disponibles -como determinados símbolos, monumentos, legislaciones, organizaciones, etc.-. Éstas no se presentan como una propuesta "mecánica", sino que se le ha otorgado otros sentidos siguiendo objetivos propios (también resignificados).

Estos movimientos y disputas por el sentido están repensando y reordenando lo político en términos culturales, debido a que "hacen el descubrimiento de las dimensiones culturales de la política, de lo político, como ámbito de producción del sentido de lo social y del reconocimiento de los diversos intereses que articulan sus conflictos y sus negociaciones" (MartínBarbero 2002: 151). En este contexto, podemos decir que los relatos históricos-políticos no están escritos de una vez para siempre, son producto de una sociedad y de su tiempo, las preguntas que se hacen están sujetas "a revisiones y refleja las tensiones políticas, culturales y sociales del momento en que se lo formula. Sin embargo, no es solamente el acuerdo sobre la efeméride histórica, sino la historia posterior la que también influye sobre esta definición" (Gutman 2012: 43).

\section{Referencias}

Fuentes Navarro, R. (2008): La comunicación desde una perspectiva sociocultural: acercamientos y provocaciones 1997-2007, Guadalajara, ITESO.

García, M. (2006a): Contar (nuestra) Mision(es). Massmediación, memoria, comunidad, identidad. En Unirevista, Vol. 1, No 3, julio (pp. 1-13) (ISSN 1809-4651). Unisinos, S. Leopoldo, Brasil: <http://www.unirevista.unisinos.br/>.

García, M. (2006b): "De los medios, su estudio, sus fines. En Estudios Regionales, Año 14, No 32 (pp. 49-70), Secretaría de Investigación y Postgrado (FHyCS-UNaM).

Gutman, (2012): Planes y expectativas de conmemoración (2000-2009) En Construir bicentenarios latinoamericanos en la era de la globalización, Buenos Aires, Infinito. pp. 37-56.

Hugues, (2008): "La evolución de los grandes sistemas tecnológicos", en Actos, actores y artefactos: sociología de la tecnología. Bernal: Universidad Nacional de Quilmes, pp.101-146

Jaquet, H. (1998): Los historiadores y la producción de fronteras: el caso de la provincia de Misiones (Argentina), en Programa Gestión de las Transformaciones Sociales (MOST) Documentos de debate N. 29, UNESCO, 1998. París, 1998. Recuperado el 7 de noviembre de 2012. Disponible en: http://unesdoc.unesco.org/images/0011/001146/114633so.pdf.

Jaquet, H. (2005): Combates por la invención de Misiones, Posadas: Editorial Universitaria.

Rodríguez. Comunicación y análisis socio-técnico: sentidos sobre la identidad local en la provincia de Misiones, Argentina 


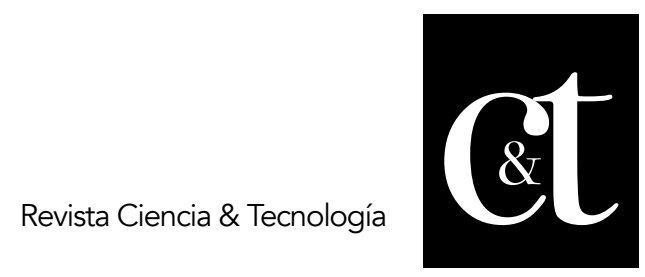

No. 30, 30 de abril de 2021

ISSN impreso: 1390 - 6321

ISSN online: 2661 - 6734

JUNTA DE ESTUDIOS HISTÓRICOS DE LA PROVINCIA DE MISIONES (2010): radas históricas sobre Misiones en el Bicentenario, Gobierno de la provincia de Misiones y la Junta de Estudios Históricos de Misiones, Posadas, Junta de Estudios Históricos de Misiones.

Machón, J. (1996): La batalla de Apóstoles y otros trabajos, Jardín América, Junta de Estudios Históricos, Sociales y Literarios de Jardín América.

Machón, J. \& Cantero, D. (2008): 1815-1821 Misiones Provincia Federal, Posadas,Editorial Universitaria de Misiones.

Machón, J. \& Cantero, D. (2010): Andrés Artigas y la Provincia Federal de Misiones. En Miradas históricas sobre Misiones en el Bicentenario, Gobierno de la provincia de Misiones y la Junta de Estudios Históricos de Misiones, Posadas, Junta de Estudios Históricos de Misiones.

Martín Barbero, J. (2002): Oficio del cartógrafo. Travesías latinoamericanas de la comunicación en la cultura, Chile, Fondo de Cultura Económica.

Rodríguez, M. I. (2016): "Los actos de memoria: un estudio sobre efemérides y actos patrios en escuelas de la provincia de Misiones (Argentina)." Tesis de Doctorado. Doctorado en Comunicación de la Facultad de Periodismo y Comunicación Social de la Universidad Nacional de Misiones. Octubre, 2016.

Thomas, H. (2008): "Estructuras cerradas versus procesos dinámicos: trayectorias y estilos de innovación y cambio tecnológico". En Actos, actores y artefactos: sociología de la tecnología, Bernal, Universidad Nacional de Quilmes, pp.217-262.

Thomas, H. \& BUCH, A. (comps.) (2008): Actos, actores y artefactos: sociología de la tecnología, Bernal, Universidad Nacional de Quilmes.

Uranga, W. (2007): "Mirar desde la comunicación. Una manera de analizar las prácticas sociales" Disponible: http://www.washingtonuranga.com.ar/images/propios/14 mirar desde.pdf 